C. Franke' ${ }^{1}$, C. Warnke ${ }^{2}$, A. Gorsler ${ }^{3}$, H. Prüß $\aleph^{4,5}$

${ }^{1}$ Klinik für Neurologie mit Experimenteller Neurologie, Charité - Universitätsmedizin Berlin, Berlin, Deutschland

${ }^{2}$ Klinik und Poliklinik für Neurologie, Medizinische Fakultät und Uniklinik Köln, Universität zu Köln, Köln, Deutschland

${ }^{3}$ Kliniken Beelitz, Beelitz-Heilstätten, Deutschland

${ }^{4}$ Klinik für Neurologie und Experimentelle Neurologie, Charité - Universitätsmedizin Berlin, Berlin, Deutschland

${ }^{5}$ Deutsches Zentrum für Neurodegenerative Erkrankungen (DZNE) Berlin, Berlin, Deutschland

\title{
Neurologische Manifestationen bei Patienten mit Post-COVID-19-Syndrom
}

\section{Definition}

- Ein Post-COVID-19-Syndrom liegt dann vor, wenn klinische Symptome und pathologische Untersuchungsbefunde während oder nach einer mit COVID-19 vereinbaren Erkrankung auftreten und für mindestens 12 Wochen nach der Akutinfektion andauern und nicht durch andere Diagnosen erklärt werden können.

- Der Begriff Long-COVID-19 umfasst klinische Symptome, die während oder nach COVID-19 auftraten; die Akutinfektion liegt hierbei mindestens 4 Wochen zurück [10].

\section{Vorkommen}

- Betroffen von neurologischen Manifestationen sind sowohl Patienten, die COVID-19 mit einem milden bis moderaten Verlauf durchmachten und in häuslicher Quarantäne verblieben, als auch Patienten, die in ein Krankenhaus aufgenommen werden mussten oder sogar intensivpflichtig behandelt wurden.

\section{Symptome}

- Neben kardiopulmonalen Symptomen wie (belastungsabhängiger) Luftnot und Herzrhythmusstörungen berichten viele der Post-COVID-19-Patienten von neurologischen und (neuro-)psychiatrischen Symptomen (vgl. Tab. 1; [1, 3, 4, 6, 16, 17]).

- Die Beschwerden können sehr unterschiedlich ausgeprägt sein, stark fluktuieren und im Wechselspiel mit anderen Stressfaktoren stehen.

- Es besteht eine verlängerte Rekonvaleszenz nach COVID-19. Eine Besserung der Residualsymptome tritt bei einer Vielzahl der Patienten ohne spezielle Behandlung in den ersten 12 Wochen nach der Akutinfektion ein.

- Bei Patienten mit intensivpflichtigem Verlauf während der Akutinfektion können die klinischen Symptome auch im Rahmen eines „post-intensive-care-syndrom“ (PICS) auftre-

\section{Redaktion}

G. Fink, Köln

J.P. Sieb, Stralsund

DGNeurologie $2021 \cdot 4$ (4): 276-280

https://doi.org/10.1007/s42451-021-00345-3

Angenommen: 27. April 2021

Online publiziert: 9 . Juni 2021

(c) Springer Medizin Verlag GmbH, ein Teil von Springer Nature 2021 ten. Beim PICS bestehen kognitive Defizite, Muskelschwäche im Sinne einer „critical illness polyneuropathy/myopathy“ (CIP/CIM), posttraumatische Belastungsstörungen (PTBS), Depressivität und Angststörungen [11, 12].

- Die Datenlage zur Post-COVID-19-Symptomatik ist aktuell noch begrenzt und erfüllt nicht konsequent die o. g. zeitlichen Post-COVID-19-Kriterien (Tab. 2). Daten zur Unterscheidung von Post-COVID-19 nach intensivpflichtigem Verlauf und PICS existieren bislang nicht.

\section{Pathophysiologie}

- Aktuell noch unbekannt. Am Beispiel der Fatigue werden neurotransmittervermittelte Veränderungen, eine postinfektiös fortbestehende Entzündung sowie (virusgetriggerte) immunvermittelte Mechanismen diskutiert [13].

- Mittels der ${ }^{18}$ FDG-PET (Fluordesoxyglukosepositronenemissionstomographie mit $\left.\left[{ }^{18} \mathrm{~F}\right]\right)$ wurde bei 10 von 15 LongCOVID-Patienten mit neurokognitivem Defizit (weniger als 26/30 Punkte im MoCA[„Montreal cognitive assessment“]Test) in frontoparietalen Hirnregionen ein Hypometabolismus nachgewiesen [5]. Beim Follow-up [2] von 8 Patienten über 6 Monate zeigte sich eine Symptomverbesserung mit weitgehender Normalisierung des Hirnstoffwechsels in der PET.

- Erfahrungen mit der SARS-1-Pandemie zeigten bereits, dass einzelne Patienten sehr lang anhaltende klinische Beschwerden zurückbehalten können, insbesondere Schmerzen, Fatigue, Depression und Schlafstörungen.

- Das Fehlen krankheitsspezifischer Biomarker erschwert die eindeutige ätiologische Zuordnung ebenso wie die Überlappung mit anderen (prämorbiden) Erkrankungen.

\section{Diagnostik}

- Eine ausführliche neurologische Untersuchung und Durchführung von symptomorientierter Zusatzdiagnostik ([15], inklusive Elektrophysiologie [EEG, ENG/EMG], Blut- und Liquoruntersuchung, neuropsychologischer Testung und Bildgebung) ist indiziert (vgl. Abb. 1) und sollte je nach klinischer Präsentation in Anlehnung an die existierenden Leitlinien der Deutschen Gesellschaft für Neurologie (DGN) erfolgen (vgl. Abb. 1).

- Eine erweiterte Diagnostik (neuropsychologische Testung und Liquoruntersuchung) bei Konzentrations- und Gedächtnisstörungen sowie Fatigue sollte erwogen werden, wenn die 
Tab. 1 Neurologische Post-COVID-19-Symptome [1-4, 15]

\begin{tabular}{|l|l|}
\hline Symptom & Häufigkeit [\%] \\
\hline Fatigue & $31-63$ \\
\hline Insomnie & $24-54$ \\
\hline Gelenkschmerzen & 27 \\
\hline Muskelschmerzen/Muskelschwäche & 23 \\
\hline Angst/Depression & $18-23$ \\
\hline Gedächtnis/Konzentrationsstörung & 21 \\
\hline Riechstörung/Geschmacksstörung & $10-15$ \\
\hline Kopfschmerzen & 10 \\
\hline Schwindel & 8 \\
\hline
\end{tabular}

Beschwerden 12 Wochen nach der Akutinfektion noch fortbestehen.

\section{Differenzialdiagnose}

- Je nach führender klinischer Präsentation müssen Erkrankungen, die im zeitlichen Zusammenhang mit COVID-19, aber nicht in kausalem Bezug dazu auftraten, ausgeschlossen werden. Dazu zählen auch Systemerkrankungen wie Tumorleiden, strukturelle Herz-/Lungenerkrankungen bzw. andere (virale) Infektionen.

- An möglichen Differenzialdiagnosen (DD) anhand der in Abb. 1 beschriebenen Symptome sind zu nennen:

- DD Neuropathie/Myalgie: u.a. Polyneuropathie, Myopathie, Myositis anderer Ätiologie [7-9]

- DD kognitive Störung/Fatigue: u.a. „chronic fatigue syndrome" (CFS), neurodegenerative Demenzen

- DD Kopfschmerzen: u.a. (chronische) Spannungskopfschmerzen

- DD Schwindel: u.a. benigner paroxysmaler Lagerungsschwindel (BPLS), Neuropathia vestibularis etc.

\section{Therapie}

- Prävention: Da eine COVID-19-Erkrankung die Voraussetzung für die Entwicklung eines Post-COVID-19 Syndroms ist, stellt die Vermeidung der Infektion den wichtigsten präventiven Faktor dar. Hierzu gehört neben allgemeinen Hygienemaßnahmen insbesondere die Impfung mit einem der verfügbaren Impfstoffe.

- Aufklärung über das Krankheitsbild, die Langzeitrisiken und Behandlungsoptionen, Unterstützung bei der Suche nach zusätzlichen psychosozialen Hilfsangeboten beispielsweise für die Wiedereingliederung in den Beruf

- Abhängig von der klinischen Präsentation erfolgt die Therapie gemäß der existierenden Leitlinien der Deutschen Gesellschaft für Neurologie (DGN): https://dgn.org/leitlinien/ll-56ll-therapie-des-episodischen-und-chronischen-kopfschmer zes-vom-spannungstyp/, https://dgn.org/leitlinien/ll-030130-therapie-akuter-und-chronischer-immunvermittelter-neuropathien-und-neuritiden-2018/, https://www.awmf.org/ leitlinien/detail/anmeldung/1/ll/017-078.html, https://dgn. org/leitlinien/leitlinie-diagnose-und-therapie-von-demenze n-2016/.

- Bei Hinweisen auf eine immunvermittelte Pathogenese in Blut oder Liquor (z. B. Pleozytose, starke Eiweißerhöhung, antineuronale oder antigliale Autoantikörper) sollte eine The-
Tab. 2 Ausgewählte Studien zu neurologischen Manifestationen Post-COVID-19

\begin{tabular}{|c|c|c|}
\hline Autor/Land & $\begin{array}{l}\text { Patienten- } \\
\text { anzahl }\end{array}$ & Hauptergebnisse \\
\hline Arnold/UK [1] & 110 & $\begin{array}{l}8-12 \text { Wochen nach Akutinfektion: } \\
3 / 4 \text { aller Patienten haben Sympto- } \\
\text { me: } 39 \% \text { Fatigue, } 23 \% \text { Muskel- } \\
\text { schmerzen, } 24 \% \text { Schlafstörungen, } \\
10 \% \text { Anosmie }\end{array}$ \\
\hline Carfi/Italien [3] & 143 & $\begin{array}{l}60 \text { Tage nach Akutinfektion: } 87 \% \\
\text { aller Patienten haben Sympto- } \\
\text { me: } 53 \% \text { Fatigue, } 27 \% \text { Gelenk- } \\
\text { schmerzen, } 15 \% \text { Anosmie, } 10 \% \\
\text { Kopfschmerzen, } 8 \% \text { Schwindel }\end{array}$ \\
\hline Chopra/USA [4] & 488 & $\begin{array}{l}60 \text { Tage nach Entlassung: } 13 \% \text { an- } \\
\text { haltende Hyposmie/Hypogeusie, } \\
49 \% \text { emotionale Beeinträchtigung } \\
\text { durch die Akutinfektion }\end{array}$ \\
\hline Tauquet/USA [16] & 62.354 & $\begin{array}{l}\text { 14-90 Tage nach Akutinfektion: } \\
\text { erhöhtes Risiko für multiple psychi- } \\
\text { atrische Erkrankungen und Demenz } \\
\text { (2- bis 3-fach erhöht) }\end{array}$ \\
\hline $\begin{array}{l}\text { "Writing com- } \\
\text { mittee for the } \\
\text { COMEBAC Study } \\
\text { Group", Frankreich } \\
\text { [17] }\end{array}$ & 487 & $\begin{array}{l}4 \text { Monate nach Entlassung: } 31 \% \\
\text { Fatigue, } 21 \% \text { kognitive Defizite, } \\
54 \% \text { Schlafstörungen; bei ITS-Pa- } \\
\text { tienten: } 23 \% \text { Angststörung, } 18 \% \\
\text { Depression }\end{array}$ \\
\hline Huang/China [6] & 1733 & $\begin{array}{l}6 \text { Monate nach Entlassung: } 63 \% \\
\text { Fatigue/Muskelschwäche, } 26 \% \\
\text { Schlafstörungen, } 23 \% \text { Angststörung } \\
\text { und Depression }\end{array}$ \\
\hline
\end{tabular}

rapie mit Glukokortikoiden, i.v. Gabe von Immunglobulinen (IVIG) oder therapeutischer Apherese in Abhängigkeit von Risiko und Nutzen erwogen werden.

- Eine interdisziplinäre Behandlung unter Einbeziehung von internistischer, psychosomatischer und psychiatrischer Expertise ist sinnvoll. Besteht führend belastungsabhängige Dyspnoe, sollte eine pulmologische Vorstellung, bei Herzrhythmusstörungen und Tachykardie eine kardiologische Vorstellung erfolgen. Bei Angststörungen, Panikattacken, Depressionen und funktionellen neurologischen Störungen ist eine psychiatrische bzw. psychosomatische (Mit-)Behandlung anzustreben.

- Bisher gibt es keine validen klinischen oder laborchemischen Parameter, die die Prognose eines Post-COVID-19-Syndroms eingrenzen lassen.

\section{Rehabilitation}

- Ziel der Rehabilitationsmaßnahmen sind eine Funktionswiederherstellung sowie die Wiederherstellung der Leistungsfähigkeit der Betroffenen in Alltag und Beruf([14], https://www. awmf.org/leitlinien/detail/ll/080-008.html).

- Bei erst später im Verlauf festgestellten Post-COVID-19Symptomen sollen nach der ärztlich diagnostischen Abklärung primär ambulante Heilmittel verordnet werden, um die eingeschränkten Körperfunktionen wiederherzustellen.

- Eine Indikation zur Frührehabilitation und Rehabilitation mit Schwerpunkt auf die Versorgung von insbesondere neurologischen, ggf. auch psychiatrischen und psychosomatischen Symptomen besteht für Patienten mit schwerem COVID-19- 


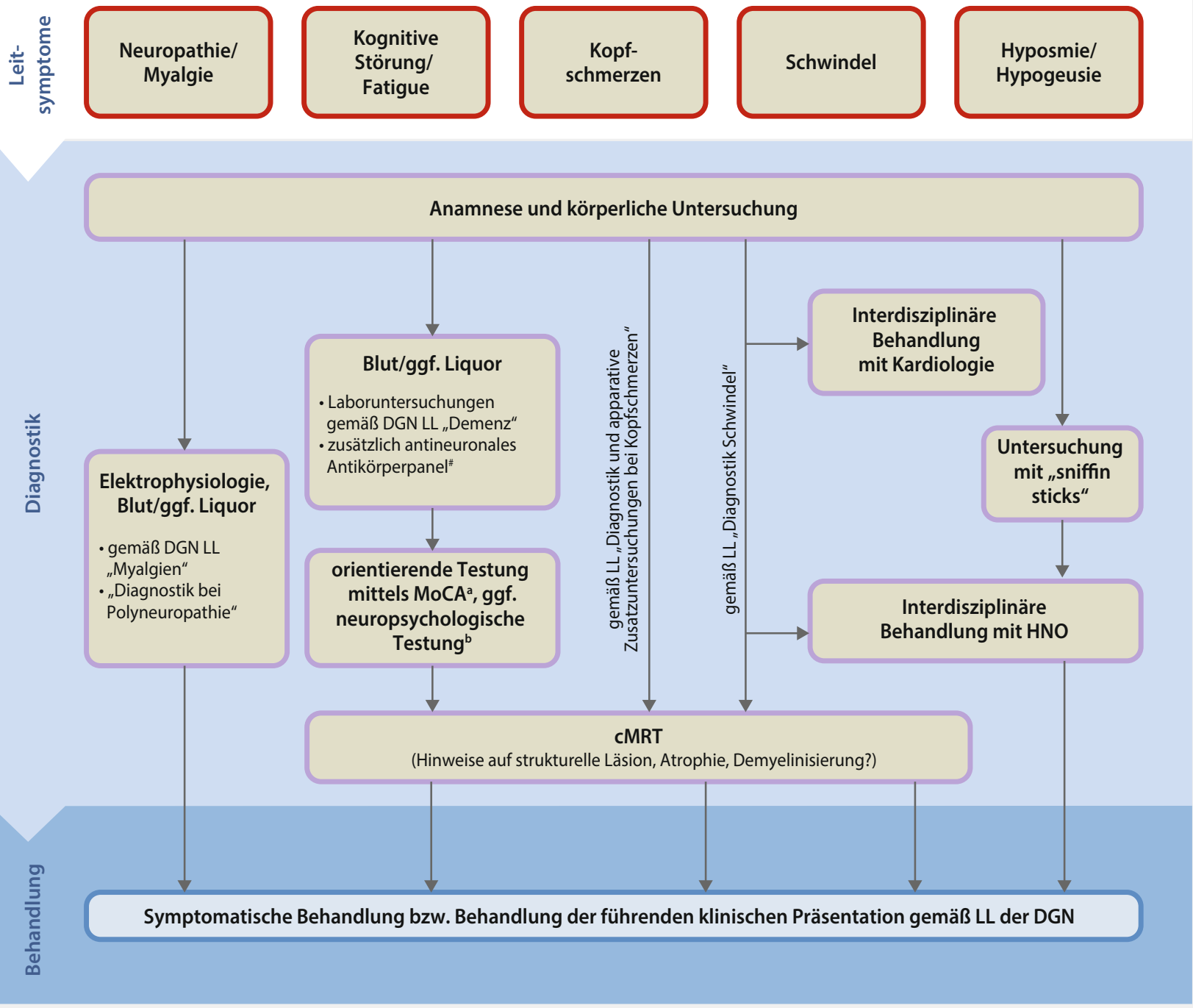

Abb. 1 SOP-Algorithmus bei neurologischen Manifestationen eines Post-COVID-19 Syndroms, CMRT kraniale Magnetresonanztomographie,

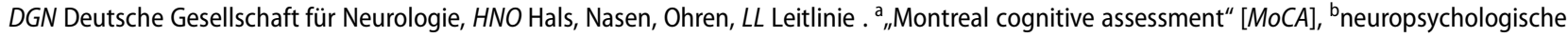
Testung: Untersuchung von Gedächtnis, Sprache, Exekutivfunktion, Aufmerksamkeit, Visuokonstruktion und figuralem Abruf, cantineuronales Antikörperpanel: nach klinischer Priorisierung

Akutverlauf direkt im Anschluss an die primäre Krankenhausbehandlung sowie nach milden und moderaten Verläufen, wenn residuelle Symptome die Teilhabe am gesellschaftlichen und Arbeitsleben relevant einschränken.

- Bei COVID-19-Patienten mit langem intensivmedizinischem Aufenthalt und invasiver Beatmung in der Akutinfektion besteht häufig eine CIP/CIM. Darüber hinaus können Enzephalopathien und Delirien mit über Wochen anhaltenden neurokognitiven Störungen des Kurz- und Langzeitgedächtnisses, der Aufmerksamkeit, der Orientierungsfähigkeit und der visuokonstruktiven Fähigkeiten sowie auch emotionalen Störungen persistieren.

- Nach Entlassung der Patienten aus der Frührehabilitation/ Rehabilitation sollten zum einen ambulant die störungsorientierte Therapie fortgesetzt werden sowie Kontrollen des Rehabilitationsfortschritts und des weiteren Rehabilitations-,
Therapie- oder psychosozialen Unterstützungsbedarfs mindestens 1-mal im Quartal im ersten Jahr nach der Akuterkrankung erfolgen.

\section{Offene Fragen}

Die systematische Betreuung der Patienten in Post-COVID-19Sprechstunden kann dazu beitragen, wichtige offene medizinische Fragen zu beantworten. Dazu gehören:

- Wie ist der natürliche Verlauf der Erkrankung und ihrer Spätfolgen?

- Lassen sich klinische, laborchemische oder bildgebende Biomarker identifizieren, die die Prognoseabschätzung erleichtern?

- Welche medikamentösen, supportiven oder psychosozialen Interventionen bewähren sich in der Praxis? 
Bei der neurologischen Diagnostik und Therapie von Komplikationen der COVID-19-Erkrankung ist vieles wissenschaftlich noch nicht gesichert. Wir danken den Autoren sehr für diese aus unserer Sicht überaus instruktive SOP. Sie ist für uns aktueller Ausgangspunkt für die weitere Entwicklung von neurologischen Standards bei der Betreuung von COVID-19-Erkrankungen. Vielleicht wollen Sie als Leser der DGNeurologie dazu beitragen und über Ihre eigenen klinischen Erfahrungen in Form eines Leserbriefs berichten.

Prof. Dr. G. Fink, Köln

Prof. Dr. J.P. Sieb, Stralsund

- Auf welche Beschwerden, im neurologischen Fachgebiet ausgerichtet, sollte ein rehabilitatives Angebot mit den Kostenträgern entwickelt werden und welche benachbarten Disziplinen sollten im interdisziplinären Setting in das Rehabilitationskonzept der Post-COVID-19-Patienten eingebunden sein?
Korrespondenzadresse

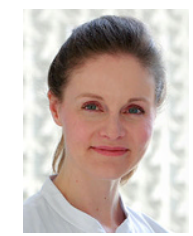

Dr. med. C. Franke

Klinik für Neurologie mit Experimenteller Neurologie

Charité - Universitätsmedizin Berlin

Hindenburgdamm 30, 12200 Berlin, Deutschland

christiana.franke@charite.de

\section{Einhaltung ethischer Richtlinien}

Interessenkonflikt. C. Franke, C. Warnke, A. Gorsler und H. Prüß geben an, dass kein Interessenkonflikt besteht.

Für diesen Beitrag wurden von den Autoren keine Studien an Menschen oder Tieren durchgeführt. Für die aufgeführten Studien gelten die jeweils dort angegebenen ethischen Richtlinien.

Literatur

1. Arnold DT, Hamilton FW, Milne A et al (2020) Patient outcomes after hospitalisation with COVID-19 and implications for follow-up: results from a prospective UK cohort. Thorax. https://doi.org/10.1136/thoraxjn-2020-216086

2. Blazhenets G, Schroter N, Bormann T et al (2021) Slow but evident recovery from neocortical dysfunction and cognitive impairment in a series of chronic COVID19 patients. J Nucl Med. https://doi.org/10.2967/jnumed. 121.262128

3. Carfi A, Bernabei R, Landi F et al (2020) Persistent symptoms in patients after acute COVID-19. JAMA 324:603-605

4. Chopra V, Flanders SA, O'malley M et al (2020) Sixty-day outcomes among patients hospitalized with COVID-19. Ann Intern Med. https://doi.org/10.7326/M20-5661

5. Hosp JA, Dressing A, Blazhenets G et al (2021) Cognitive impairment and altered cerebral glucose metabolism in the subacute stage of COVID-19. Brain. https://doi. org/10.1093/brain/awab009

6. Huang C, Huang L, Wang Y et al (2021) 6-month consequences of COVID-19 in patients discharged from hospital: a cohort study. Lancet 397:220-232

7. https://dgn.org/leitlinien/II-030-051-diagnostik-und-differenzialdiagnose-beimyalgien-2020/.Zugegriffen: 27. Mai 2021

8. https://dgn.org/leitlinien/II-030-130-therapie-akuter-und-chronischerimmunvermittelter-neuropathien-und-neuritiden-2018/.Zugegriffen: 27. Mai 2021

9. https://dgn.org/leitlinien/II-69-II-myositissyndrome/.Zugegriffen: 27. Mai 2021

10. https://www.nice.org.uk/guidance/ng188. Zugegriffen:27. Mai 2021

11. Labuzetta JN, Rosand J, Vranceanu AM (2019) Review: post-intensive care syndrome: unique challenges in the neurointensive care unit. Neurocrit Care 31:534-545

12. Marra A, Pandharipande PP, Girard TD et al (2018) Co-occurrence of post-intensive care syndrome problems among 406 survivors of critical illness. Crit Care Med 46:1393-1401

13. Rudroff T, Fietsam AC, Deters JR et al (2020) Post-COVID-19 fatigue: potential contributing factors. Brain Sci. https://doi.org/10.3390/brainsci10121012

14. Schlitt A, Schultz K, Platz T (2021) AWMF-Leitlinie: Rehabilitation nach einer COVID19-Erkrankung. Dtsch Arzteb/ 118(15):A-774 / B-648

15. Schweitzer F, Kleineberg NN, Goreci Y et al (2021) Neuro-COVID-19 is more than anosmia: clinical presentation, neurodiagnostics, therapies, and prognosis. Curr Opin Neurol. https://doi.org/10.1097/WCO.0000000000000930

16. Taquet M, Luciano S, Geddes JR et al (2021) Bidirectional associations between COVID-19 and psychiatric disorder: retrospective cohort studies of 62354 COVID19 cases in the USA. Lancet Psychiatry 8:130-140

17. Writing Committee for The CSG, Morin L, Savale L et al (2021) Four-month clinical status of a cohort of patients after hospitalization for COVID-19. JAMA. https:// doi org/10.1001/jama.2021.3331 
Hier steht eine Anzeige.

Springer 